\title{
Multiterminal DC grids: Operating analogies to AC power systems
}

\author{
Kumars Rouzbehi $^{\mathrm{a}, *}$, J. Ignacio Candela ${ }^{\mathrm{a}}$, Gevork B. Gharehpetian ${ }^{\mathrm{b}}$, Lennart Harnefors ${ }^{\mathrm{c}}$, \\ Alvaro Luna $^{\mathrm{a}}$, Pedro Rodriguez ${ }^{\mathrm{a}, \mathrm{d}}$ \\ a Technical University of Catalonia, Barcelona, Spain \\ b Amirkabir University of Technology, Tehran, Iran \\ c ABB Power Systems, Ludvika, Sweden \\ d Abengoa, Abengoa Research, Seville, Spain
}

\section{A R T I C L E I N F O}

\section{Keywords:}

AC-DC power systems

Multi-terminal DC (MTDC) grids

MTDC grid operation and control

\begin{abstract}
A B S T R A C T
Nowadays, some Multi-terminal DC (MTDC) systems are in operation around the world. Soon, MTDC grids will be built and overlay the present AC grids. The main driver for the construction of such a grid is to facilitate large-scale integration of remote renewable energy sources to existing AC grids and to develop the energy market.

This paper presents a comprehensive analogy between the control and operation aspects of the emerging MTDC grids to those of the traditional AC power grids. Similarities and difference between the two technologies are presented and highlighted. Based on the performed detailed overview, even though a three-layered control system, i.e., primary, secondary, and tertiary control layers is state-of-the-art in large-scale AC power systems, a two-layered control system will satisfy MTDC grids control and operation requirements. This paper also addresses some control and operational issues and limitations of MTDC grids.
\end{abstract}

\section{Introduction}

In the "war of currents" in the late 1880s Edison and Westinghouse publicly battled over DC versus AC [1]. This debate was settled at the end of the 19th century as AC with frequency of $50 \mathrm{~Hz}$ or $60 \mathrm{~Hz}$ became the dominant way of power transmission and distribution. In fact, $\mathrm{AC}$ won owing to the ability of stepping up the voltage to tens or hundreds of kilovolts using transformers. This allowed low-loss bulk-power transmission from centralized power stations even over long distances.

One shortcoming of $\mathrm{AC}$ is that long-distance subsea transmissions generally are infeasible, owing to a large per-kilometer cable capacitance. This problem spurred the revival of DC, which began in 1954, when ABB's predecessor ASEA connected the Gotland Island to Sweden mainland with a $100 \mathrm{kV}$ High Voltage DC (HVDC) link [2]. This link, which utilized mercury-arc valves, was in 1970 re-engineered for $150 \mathrm{kV}$ as the first thyristor-based HVDC transmission.

Presently, the number of operational HVDC transmissions worldwide approaches to 180 . Although some of these are of point-to-point type, linking two asynchronous AC power systems, most bridge long distances, i.e., hundreds or even thousands of kilometers. For bulk transmission, e.g., from remote hydro resources, HVDC is more efficient than AC, even for overland transmission, due to lower losses, reduced transmission-line right-of-way requirements and smaller towers, and/or the possibility of undergrounding [1].
As a result, many electric power systems today feature a combination of AC and HVDC transmissions. With the increasing desire to replace the thermal generation using fossil fuel and nuclear with renewable, mainly hydro, wind, and solar [3] the attractiveness of HVDC transmission increases. This is because.

1) Hydro and wind resources often are remote to urban centers,

2) Wind and solar unlike hydro, are uncontrollable resource, which require improved transmission for proper balancing,

3) Power electronic devices develop at a rapid pace, with falling prices and increased ratings.

One existing case is the German energy transition, where nuclear generation is being phased out and replaced with renewables. In this process, a number of north-south AC transmission lines will be converted to HVDC, allowing increased power transmission without any increase of the transmission-line corridor [4].

With the exception of a few more than two-terminal HVDC transmissions system, all currently operational HVDC transmissions are two-terminal point-to-point links. However, the aforementioned drivers eventually will result in the construction of High-Voltage MultiTerminal DC (HV-MTDC) grids, interconnecting and overlaying the present AC grids that is illustrated in Fig. 1. The interconnection of North Sea offshore wind parks with Scandinavian hydropower sources 


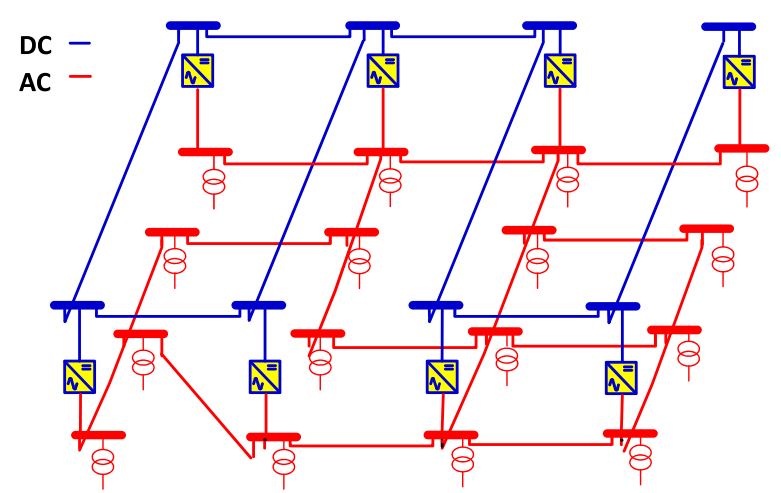

Fig. 1. MTDC grid typical representation.

and Mediterranean solar generations are the most important drivers for MTDC grids development [4].

In the longer term, the goal is to develop the "European Supergrid" with MTDC grid as its backbone, which is an activity vigorously promoted by the European Union [8].

Classic Thyristor-based HVDC transmission, rated at $\pm 800 \mathrm{kV}$ direct voltage was cutting age until 1999, when Voltage Source Converter (VSC) based HVDC emerged as an alternative [1,12]. In this year for the first time in the world, in Gotland a commercial VSC based HVDC system commissioned to deliver power from wind generation.

As VSC-HVDC allows control in not only the turn-on instant of the valves but also the turn-off instant, alternating-voltage or reactivepower control is facilitated as is important in cases of the connection to weak or passive networks. In turn, this facilitates the connection of large offshore wind farms [2-4]. Further benefit of VSC HVDC is that, unlike the classical HVDC, direct-voltage polarity change is not required for power reversal. For this reason, the VSC technology is the predominant power converter type for MTDC grid proposals.

MTDC grids have been an attractive research field in recent last years in both academia and industry. So far, several papers in the topic of MTDC grids control and operation have been published [5-11]. Yet, it can be said that the collective body of knowledge in control and operation of AC power systems is hugely larger, given that these have been around for more than a century and have been the focus of intensive research during much of that period.

In the light thereof, this study attempts to adapt the experiences gathered from the context of AC power systems to MTDC grids by pointing out their similarities and differences as well as MTDC grids control strategy inspired by well-known AC power systems control framework.

During the presentation of the paper's materials, significant amount of papers supporting the presented philosophy are reviewed and discussed.

This paper is structured as follows. Section 2 presents the analogies between AC and DC in terms of their parameters. Similarities and difference of AC and DC power grids are discussed in Section 3. MTDC grids hierarchical control structure inspired by AC power systems is proposed in Section 4. An automation pyramid for control of MTDC grids is proposed in Section 5. Conclusions of the study are presented in Section 6.

\section{Analogies between AC and DC parameters and characteristics}

With more penetration of the renewable energy resources, the AC power systems are experiencing a variety of generating units: the synchronous generators (SG) are being combined with various renewable resources interfaced by power converters. At the beginning of 2016, the installed wind and solar energy in the EU countries reached to capacity of $141.579 \mathrm{GW}$ and $95.350 \mathrm{GW}$ respectively, which corre- sponds to $15.6 \%$ and $10.5 \%$ of the total European electricity generation capacity. Renewable resources have increased their share from $24 \%$ of total power capacity in 2000 to $44 \%$ at the beginning of 2016 [3].

Although the behavior of the power systems still is dictated by large synchronous generators, but it will significantly change in the near future by high penetration rate of renewables. In recent years, mainly two movements have been started among the academia and industry pioneers. The first movement imposes new requirements for the integration of renewable resources to the power systems. As explained in [6], new renewable resources installations must present the same characteristics of synchronous generator, including, virtual inertia emulation, voltage control and frequency support [18]. The second movement states that for handling of high penetration of renewable resources they must be integrated into the MTDC grids, which will be work in pair with the existing power systems [4-7].

Various DC resources such as solar, fuel cells, batteries as well as $\mathrm{AC}$ resources such as wind generators and synchronous generators can be connected into MTDC grid together through their corresponding $\mathrm{DC} / \mathrm{DC}$ and AC/DC power converters [10-16]. MTDC grids will have various links to several AC grids through proper bidirectional DC/AC power converters to realize a large AC-DC grid [17-21].

The following sub-sections presents comprehensive analogy between $\mathrm{AC}$ and DC systems to understand how these two systems can work together.

\subsection{Analogy in terms of synchronization parameter}

Frequency is the synchronizing parameter of AC grids while in MTDC grids based on a close analogy, the direct voltage can be considered as the synchronization parameter. In the AC-DC networks, the interfacing converters would have to behave as "interpreter" translating $\mathrm{AC}$-side frequency deviations into proper DC-side voltage variations on the and vice-versa.

An inspirational understanding of the role of the interfacing power converters can be obtained by the physical example illustrated by Fig. 2. Let us consider a mechanically coupled DC motor and a synchronous generator simplified model as well as a load at the synchronous generator terminals. It can be shown through following equations that by changing the DC motor terminal feeding voltage, the output AC voltage frequency of the synchronous generator will change accordingly as showed by the following equations [22]:

$E_{a}=k \phi n$

$V_{t}-R_{a} I_{a}=E_{a}$

where $E_{a}$ is DC motor Electromotive Force (EMF), $k$ is a constant related to design of machine, $n$ is the rotation speed, $V_{t}$ is the terminal voltage and $I_{a}$ is the armature current. The magnetic flux $\phi$ is considered constant. Rotation speed of the synchronous generator is ex-

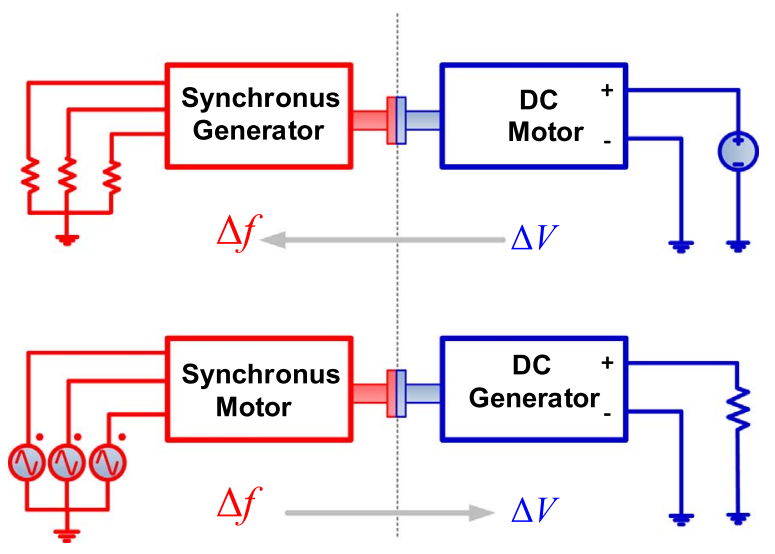

Fig. 2. Illustration of analogy between $V$ and $f$. 
pressed by the following equation:

$n=120 \frac{f}{p}$

here $f$ is the frequency and $p$ is the number of poles of the synchronous generator [22].

Now, let us consider next scenario, where the synchronous machine operates as a motor and the DC machine as a generator. Here, the synchronous motor is supplied by a variable frequency AC source, and DC generator is loaded by a resistor, then by changing the AC source frequency, the direct voltage at the DC terminal will change accordingly.

The interface power converter for AC-DC grid should behave as presented two-machine system. The frequency and the direct voltage show the energy state of the network, as changing the grid frequency or DC voltage indicates a difference between the generated power and demand.

\subsection{Analogy in terms of active power transfer}

Steady-state flow of power in an HVDC transmission line from DC bus $j$ to $k$ of Fig. 3 can easily be derived by,

$P_{j k}=\frac{V_{j}\left(V_{j}-V_{k}\right)}{R_{j k}}=\frac{V_{j} \Delta V_{j k}}{R_{j k}}$

where $V_{j}$ and $V_{k}$ are the direct voltages at buses $j$ and $k$, respectively, while $R_{j k}$ indicating the resistance of the conductor connecting two busses. Note that (4) indicates the power at the sending end.

On the other hand, transferred power in the steady state condition from bus $j$ to bus $k$ of Fig. 3 can be calculated by:

$P_{j k}=\frac{V_{j} V_{k} \sin \delta_{j k}}{X_{j k}}$

where $\delta_{j k}$ is the phase angle difference of $V_{j}$ and $V_{k}$ phasors and $X_{j k}$ represents the line reactance between buses $j$ and $k$.

By comparing (4) and (5) active power transfer analogy between DC and $\mathrm{AC}$ grids become clear.

\subsection{Analogy in terms of system energy and inertia}

Another analogy can be drawn considering the synchronous generating units inertia of AC grids and the stored energy in capacitors of DC grids, as they present the storage capacity for power changes in case of transients.

In case of any difference between the input mechanical power and the output electrical power of a synchronous generating unit, the generator rotational speed will change considering the presence of the
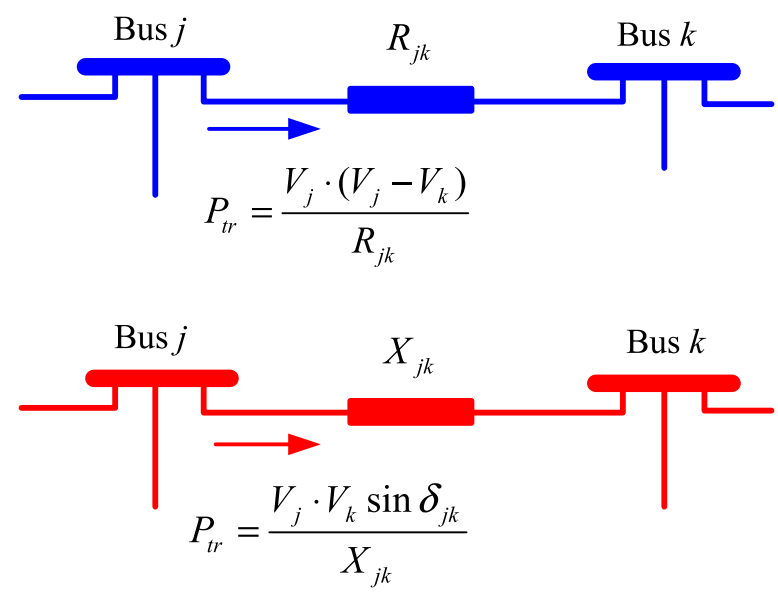

Fig. 3. AC and DC Power transfer. rotating inertia $J$.

Frequency and rotational speed are directly related to each other in AC power systems. The total stored Kinetic Energy (KE) is equal to:

$K E=\sum_{p=1}^{N} \frac{1}{2} J_{p} \omega^{2}$

where $J_{p}$ is the moment of inertia of the $p$-th synchronous machine rotating mass, $N$ indicates number of the rotating machines available in the power system.

In MTDC networks, the balancing energy will be obtained by the stored energy in the grid capacitors.

$C E=\sum_{p=1}^{N_{c}} \frac{1}{2} C_{p} V_{d c, p}^{2}$

where $N_{C}$ is the number of capacitors of the MTDC grid.

An analogous energy equation is obtained. Except that opposed to the frequency, the bus direct voltage is not a global parameter. Difference between input and output power of the whole capacitors is balanced by a change in the $C E$. Therefore, the MTDC grid capacitors $\left(C_{p}\right)$ have the same function of the rotating inertia $\left(J_{p}\right)$ of $\mathrm{AC}$ grid.

\section{4. $P / f$ and $P / V$ analogy and droop control concept}

In AC power systems, the swing equation is expressed by [23]:

$J \omega_{m} \frac{d \omega_{m}}{d t}=P_{m}-P_{e}$

where $\omega_{m}$ indicates mechanical rotational speed of the synchronous generator and $P_{m}$ and $P_{e}$ represent the mechanical input and electrical output power of the synchronous generators, respectively. Change in frequency indicate differences between $P_{m}$ and $P_{e}$, and will be balanced by change in the grid KE.

The frequency dynamics of a synchronous generator can be presented by:

$\frac{d \omega_{e}}{d t}=\frac{\omega_{e 0}^{2}}{2 S_{B} H_{a c} \omega_{e}}\left(P_{m}-P_{e}\right)$

where $S_{B}$ is the nominal power of the synchronous generating unit, $\omega_{e}$ is the electrical rotational speed of the machine and 0 subscript indicate the nominal conditions. Moreover, the term $H_{a c}$, expressed in seconds, is known as synchronous machine constant of inertia of and defined by:

$H_{a c}=\frac{0.5 J \omega_{m 0}^{2}}{S_{B}}$

The block diagram representation of (9) is illustrated in Fig. 4[23].

By linearizing of this non-linear equation around the nominal operating point, and substituting the angular velocity with the frequency, then:

$\Delta \dot{f}=\frac{f_{0}}{2 S_{B} H_{a c}}\left(\Delta P_{m}-\Delta P_{e}\right)$

From (11), by controlling the mechanical input power of the system we can control the frequency of the system.

Most approved selection for the AC power systems primary control level is the power-frequency $(P / f)$ droop control. This control is simply a proportional feedback controller that links the deviations of fre-

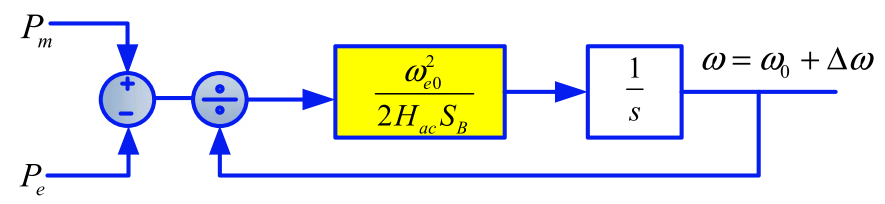

Fig. 4. Frequency dynamics block diagram representation of AC system. 


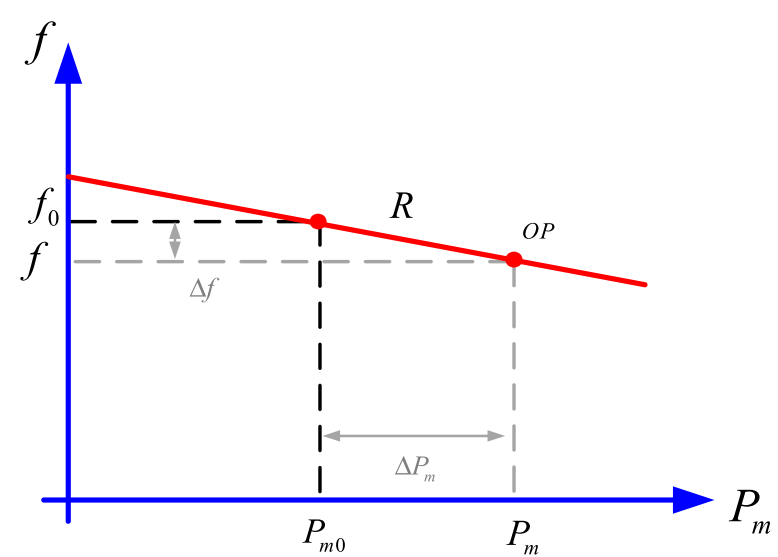

Fig. 5. A typical $P / f$ droop characteristic in AC systems.

quency to deviations of the mechanical power as follows:

$P_{m}-P_{m 0}=\frac{1}{R}\left(f-f_{0}\right)$

here $R$ is the slope of the $P / f$ characteristic as is showed in Fig. 5 .

An analogues version can be found by starting from the equation of the capacitor voltage (13) while keeping the terms $P_{m}$ and $P_{e}$ to emphasis on the energy flowing in and out of the capacitor.

$C V_{d c} \frac{d V_{d c}}{d t}=P_{m}-P_{e}$

here $C$ is the capacitance of the capacitor and $V_{d c}$ indicates the voltage in the capacitor terminals. Then, by applying the same explained mathematical procedure, the DC-bus voltage non-linear dynamics can be expressed as:

$\frac{d V_{d c}}{d t}=\frac{V_{d c 0}^{2}}{2 S_{B} H_{d c} V_{d c}}\left(P_{m}-P_{e}\right)$

where

$H_{d c}=\frac{0.5 C V_{d c 0}^{2}}{S_{B}}$

The $H_{d c}$ in (15) is not a well-established concept, but by comparing (15) and (10) the definition of "constant of inertia for the DC-link capacitor" seems sensible.

At this moment, the analogy exist between (8) and (13) as well as between (9) and (14) come to be clear.

Generally, for VSC stations, $H_{d c}$ is in the order of $10 \mathrm{~ms}$ and it is much less than conventional AC units, which have inertia constants $H_{a c}$ between $2 \mathrm{~s}$ and $10 \mathrm{~s}$ [24].

Similarly, the block diagram representation of Fig. 6 can be obtained to highlight the correspondence between the power-frequency dynamics and power-voltage dynamics.

Following the same procedure of the AC system, if the non-linear transfer function of (14) linearize around its nominal operating point, the linearized voltage deviation in terms of power deviations can be expressed as:

$\Delta \dot{V}_{d c}=\frac{V_{d c 0}}{2 H_{d c} S_{B}}\left(\Delta P_{m}-\Delta P_{e}\right)$

Since the two systems are behaving similar to each other, it seems

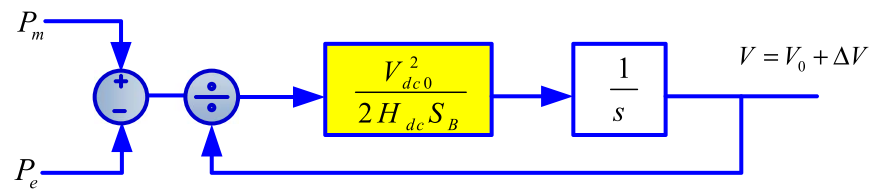

Fig. 6. Voltage dynamics block diagram representation DC system.

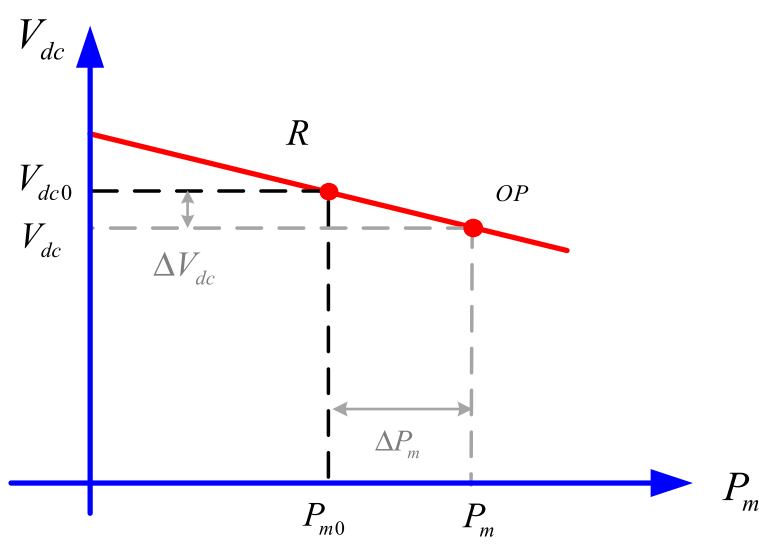

Fig. 7. Proposal of $P / V$ droop in DC systems.

logical to apply the same control strategy for both systems. Trying to develop the $P / f$ droop concept for DC systems will yield to $P / V$ droop characteristic as illustrated in Fig. $7[8,25,26,40]$.

The control law presented in (17), enables the parallel connection of similar devices as in the case of AC power systems.

$P_{m}-P_{m 0}=\frac{1}{R}\left(V_{d c}-V_{d c 0}\right)$

The $P / f$ droop characteristic typically spans from zero to nominal power because the generating units are not designed to allow passing from generator mode to motor. However, in the DC systems, units such as batteries or power converters at the connection point with AC-grids should allow bi-directional power flow. In this case, the $P / V$ droop should span from negative nominal power, to positive nominal power on the horizontal axis $[17,39]$.

The correspondence between the parameters of the two AC and DC power systems are summarized in Table 1 . The main difference between $\mathrm{AC}$ frequency control and direct voltage control is the time scale of the direct voltage variations, which is a couple of orders smaller than its AC frequency counterpart.

Control of DC voltage becomes more challenging, when taking into account the fact that the direct voltage at the different buses differs as a result of the power flows through the lines.

\section{Similarities and differences of AC and MTDC grids control and operation}

Control of VSC stations in MTDC grids would be very similar to control of synchronous generators in AC grid. This similarity is illustrated by Fig. 8. While for an AC turbo-generator system, the governor and exciter are in charge of control of frequency (or active power) and $\mathrm{AC}$ voltage (or reactive power) [30], similar arrangement can be established for a DC system, where the active channel of the VSC controls the active power or frequency and the reactive channel

Table 1

Correspondence between AC and DC parameters.

\begin{tabular}{lll}
\hline Characteristic & AC grid & DC grid \\
\hline Grid state of energy & Target frequency $(\omega)$ & Target DC voltage $\left(V_{d c}\right)$ \\
Voltage change & $V \cdot \sin \delta$ & $\Delta V_{d c}$ \\
Impedance & $X_{a c}$ & $R_{d c}$ \\
Active power & $\left(V_{1} V_{2} \sin \delta\right) / X_{a c}$ & $\left(V_{d c} \Delta V_{d c}\right) / R_{d c}$ \\
Inertia & $J$ & $C$ \\
Energy & $J \omega^{2} / 2$ & $C V_{d c}^{2} / 2$ \\
\hline
\end{tabular}




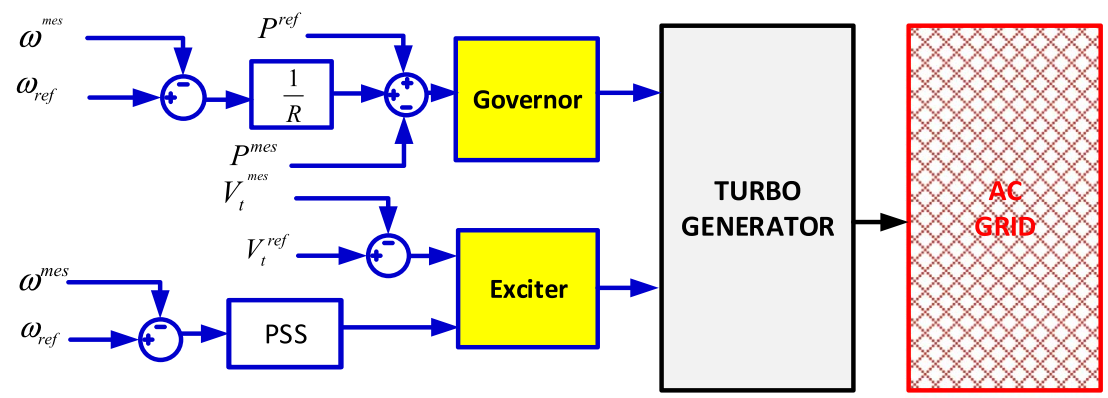

Synchronous Generator Control in AC Grid.

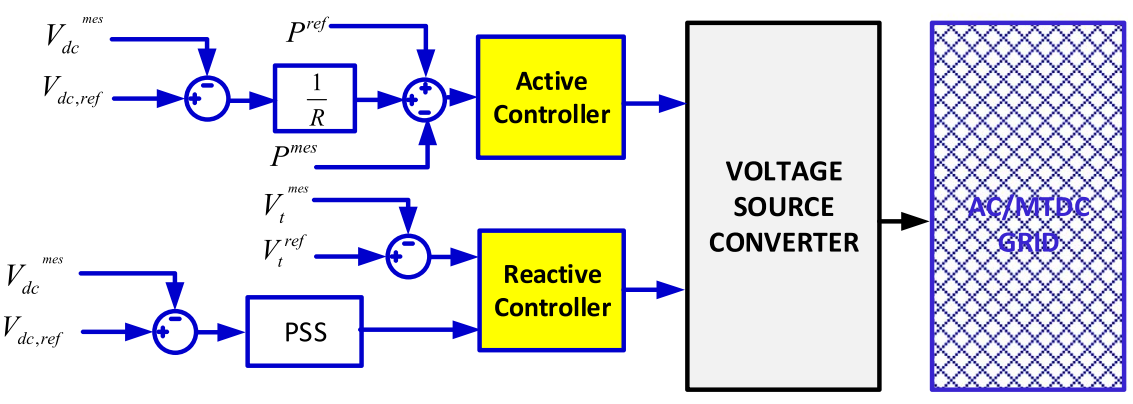

Voltage Source Converter Control in AC/MTDC Grid.

Fig. 8. Similarities between control of SG and VSC.

controls AC voltage or reactive power [17].

The presented analogies in the previous section can be employed to highlight the similarities as well as difference of AC and MTDC grids control and operation.

The most fundamental differences of $\mathrm{AC}$ and $\mathrm{DC}$ grids originate from two issues, the energy storing elements (i.e., capacitors and inductors) and frequency. In MTDC grid, owing to the inactive behavior of inductors and capacitor during the steady-state conditions, there is no reactive power. Hence, the associated issues with the reactive power, as those present in $\mathrm{AC}$ systems, are not available in DC environments.

Although, thanks to such a difference the total capacity of the transmission lines will utilize for transferring active power instead of active plus reactive power. Besides, MTDC grids do not need to deal with the issues of three-phase balance, nor require assisting compensating devices to solve power quality problem.

In the AC grids, active power is directly related to network frequency and reactive power is directly related to AC voltage [31]. It will be main cause of reduction in the dynamic stability margin. Instead, in DC network active power is related only to the direct voltage and it will increase in dynamic stability margin.

While frequency and DC voltage are related to control of active power, but there are fundamental differences in control of frequency and direct voltage, as outlined below,

- Frequency in $\mathrm{AC}$ systems is a global parameter throughout the system, while direct voltage is different at different buses of a DC system.

- While it is desirable to maintain frequency at the reference value throughout AC systems, but is not desirable at all in case of direct voltage. If direct voltages of all DC buses are the same, there will not be any power flow within the DC lines.

Hence, it can be concluded that although a secondary control layer enforces frequency deviation from the nominal value to zero in ac power systems, there is no need to such control action in MTDC grids. This would lead to a simpler control framework for MTDC grids compared to AC grids, as explained in the next section.

\section{AC power system-inspired control of mtde grids}

\subsection{Brief overview of hierarchical AC power systems three-layer} control structure

AC power systems control framework is composed of a hierarchical three-layer structure divided into primary, secondary and tertiary layers [30]. In the primary control layer of the AC power systems, the governors are in charge of consumed and generated power balance. In fact, this control layer is implemented on the governor system of each synchronous generator, participating in the frequency control task, and is referred to as a $P / f$ droop control, as discussed in the previous section. Fig. 9 shows the block diagram representation of primary frequency control in $\mathrm{AC}$ grid.

The secondary control layer of AC grids modify the set point of the governors in order to guarantee that the steady state frequency will

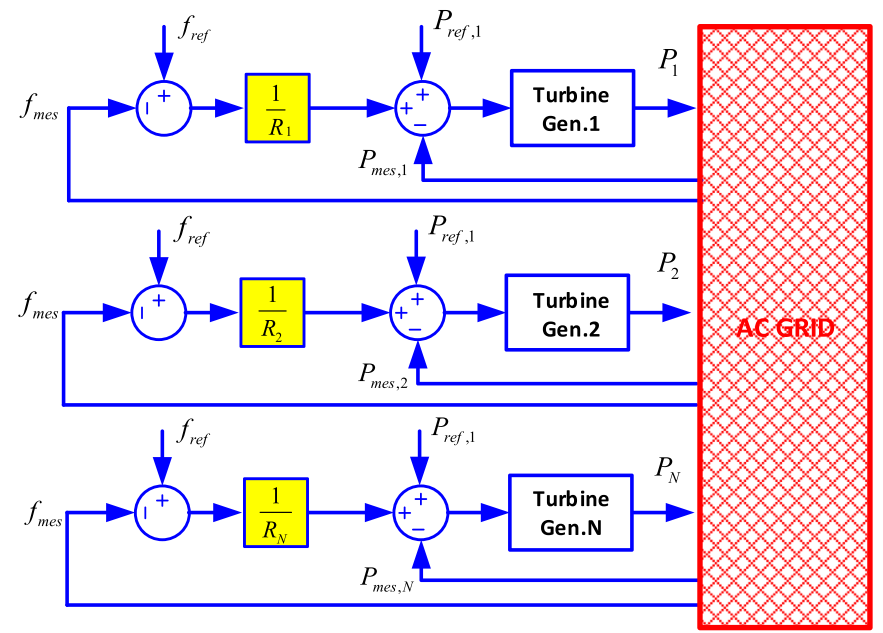

Fig. 9. AC grid primary frequency control. 


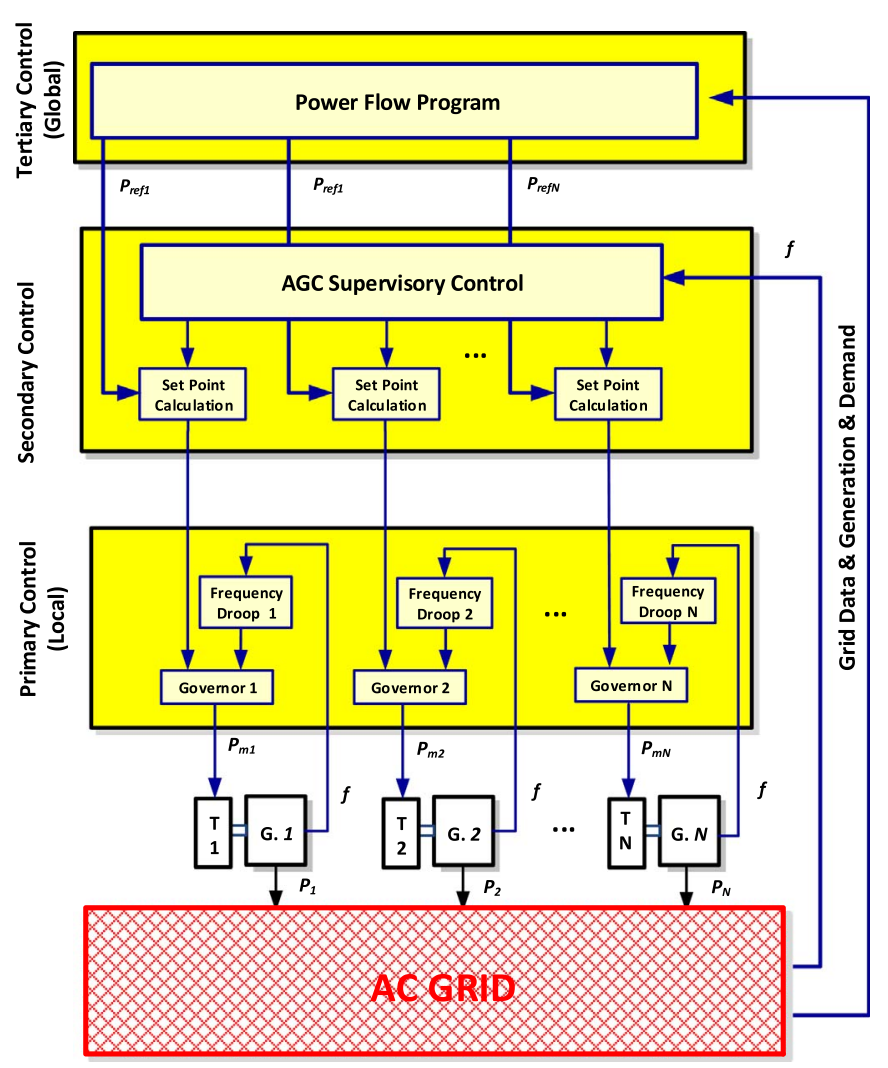

Fig. 10. AC grid three layer control structure.

always return to the specified value [31]. The governors sense any change in the rotation speed of the shaft (or grid frequency) and regulates the mechanical input power of the generator.

The secondary layer, also referred as the automatic generation control (AGC), compensates the frequency deviations, and suitably allocates the output power of generators to control the active power interchanged among transmission lines [31]. The link between primary and secondary control layers is illustrated in the three-layer control structure of Fig. 10.

Tertiary control layer acts as the upper control level of secondary and primary and schedules the proper signals, according to a power flow routine or an optimal power flow procedure, given objective function considering economics and technical aspects of the system and system constraints [31].

\subsection{Hierarchical control structure of mtdc grids: why two-layer control system?}

While a three-layer control structure is needed for AC power systems, but this is not the case for MTDC grids. Fundamental difference between $\mathrm{AC}$ and $\mathrm{DC}$ grid is absence of frequency (as a global parameter) in DC grids. Although the deviation of frequency as the synchronization parameter, throughout the AC grid must also be always forced to zero to balance generation and demand, but the deviation of direct voltage, as the synchronization parameter of MTDC grids, cannot be always maintained at a fixed reference value throughout DC grid.

In MTDC grids, functionalities like control of power flow between areas should be done by similar to AGC's in the AC grids.

The direct voltages within the grid can simply vary while the balance between generation and demand in MTDC grid is maintained. Hence, the control structure in MTDC grids should be reduced to a two-layer control system divided into a primary layer (or low level) control and a secondary layer (or high level) control.

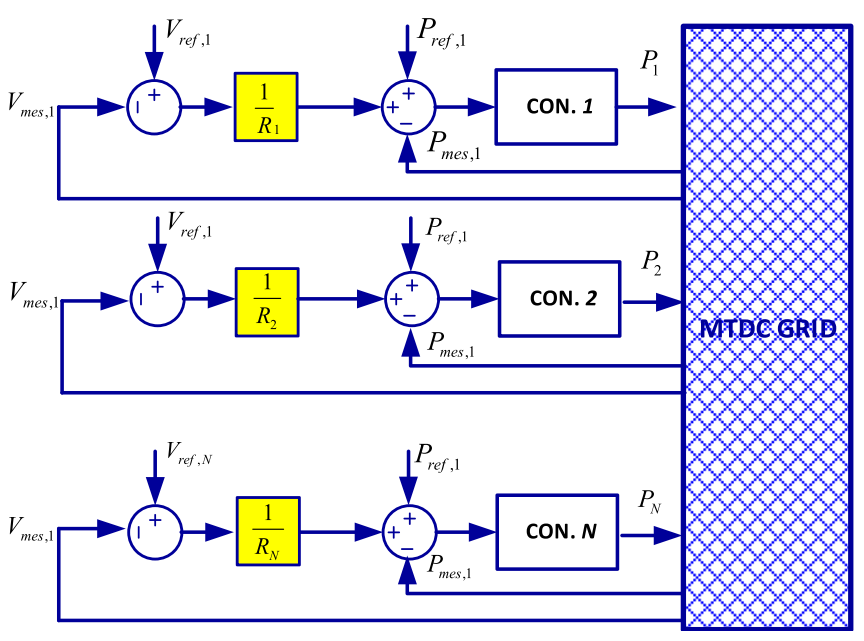

Fig. 11. Primary voltage control in MTDC grid.

\section{3. mtdc grids Low level control}

VSC shows remarkable control capabilities such as independent reactive and active power control and no need to change of the voltage polarity in case of reversal power flow [32]. The controller of each VSC station handles the received set points (i.e., controlling the voltages and powers at the $\mathrm{DC}$ and $\mathrm{AC}$ terminals with the response time in the range of tens of milliseconds). The control goals can be adjusting of a certain active or reactive power flow, frequency or direct voltage control. To participate in the power-sharing task in MTDC grid, the VSC stations adopt voltage-droop characteristic, similar to the power-frequency droop control in AC grids. This allows sharing of power in MTDC grids among several VSC stations, as shown in Fig. 11.

Generally, the VSC control does not depend on external communication. Mutual coordination among the VSC stations and the MTDC and $\mathrm{AC}$ grids can be achieved by applying scheduled characteristics, such as fixed reference set points or voltage droop characteristics. Nevertheless, the VSC stations send status and measured signals to the high-level controller and receive its control characteristics and reference values.

The most popular control technique for VSC stations in HVDC systems is decoupled vector current control [27]. In this control strategy, the VSC three-phase voltages and currents are transformed to the rotating direct-quadrature $(d q)$ reference frame that will be synchronized with AC grid voltage by use of a phase-locked loop (PLL). This low level control system determine the VSC voltage reference in the $d q$ axes through an inner-current controller, and this signal will be fed back to VSC after transforming it back to the three-phase $a b c$ reference frame [27]. A well-designed VSC inner current controller, with a high control bandwidth, significantly improves the VSC response by providing damping effect [28]. Besides, it helps in steady state to eliminate the cross-coupled interactions between the $d$ and $q$-axes to reduce the effect of the grid-side voltage variations on VSC current $[29,35]$. The VSC-HVDC vector current control structure is shown in Fig. 12.

In [34], a Particle Swarm Optimization (PSO) based algorithm is presented for optimal tuning of MTDC grid VSC stations control parameters. In addition, the voltage droop control is utilized to ensure the active power balance within the MTDC grid.

Besides, one trend is to make the VSC station possess the grid synchronization mechanisms similar to synchronous generators in order to overcome some of the difficulties of the vector-current control, for instance when the VSC station is connected to weak AC grid. This approach, known as power-synchronization control [38], allows the VSC-HVDC to maintain stable operation with higher load angles. As a 


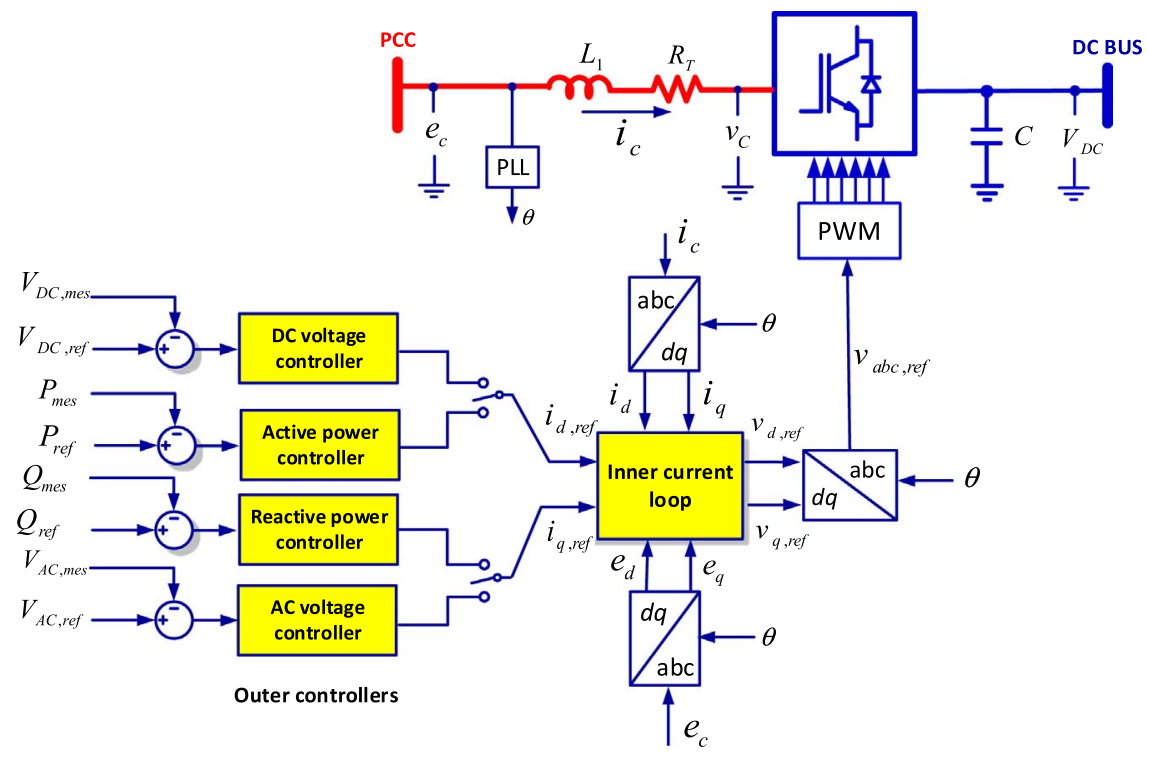

Fig. 12. VSC-HVDC vector current control structure.

result, the power converter is then able to exchange more power with $\mathrm{AC}$ grid. The VSC maximum transferable power is an important criterion for control design and stability analysis [33].

Regardless of the control technique implemented in the primary level, if the VSC stations are equipped with $P / V$ droop controllers, the balance between generation and demand can be guaranteed by the primary control level action.

\section{4. mtdc grid high level control}

MTDC grids high-level control have objective similar to the tertiary control level of AC grids. In the high-level control system of the MTDC grids, AC/DC power flow or optimal AC/DC power flow is executed considering the state and the requirements of the grid (e.g., available generation and the level of demand). Then the set points of the controllers in the primary level (e.g., parameters of the $P / V$ droop characteristics) are derived based on the power flow results [36]. In brief, for the MTDC grids and in steady state condition, the main goals of the high level control or secondary control layer, are:

- To correct and maintain the power interchange all over the grid,

- To maintain all the power flows and bus voltages in the scheduled limits.

Similar to AC grid control, the higher-level control always acts slower than lower control layer. MTDC grid hierarchical control structure is illustrated in Fig. 13. In MTDC grid, all DC bus voltages must be monitored, in contrast to the AC grids where frequency is a global and solely monitored parameter. Moreover, $P / V$ droop is implemented on voltage-regulating converters.

A DC voltage and power-sharing control structure for MTDC grids based on an optimal power flow method and voltage-droop control is presented in [36]. In the proposed approach, optimal power flow algorithm is executed at the high control level of the MTDC grid to find the optimal reference values for the DC voltages and active power of the VSC stations. Then, at the primary control level, the voltage-droop characteristics of the VSC stations are tuned based upon the optimal power flow results.

\section{5. mtdc grids VSC control requirements and constraints}

For successful operation of MTDC grid, a number of issues such as grid start-up, black start requirements, plug-and-play feature, and autonomous control should be addressed. These issues are further explained below.

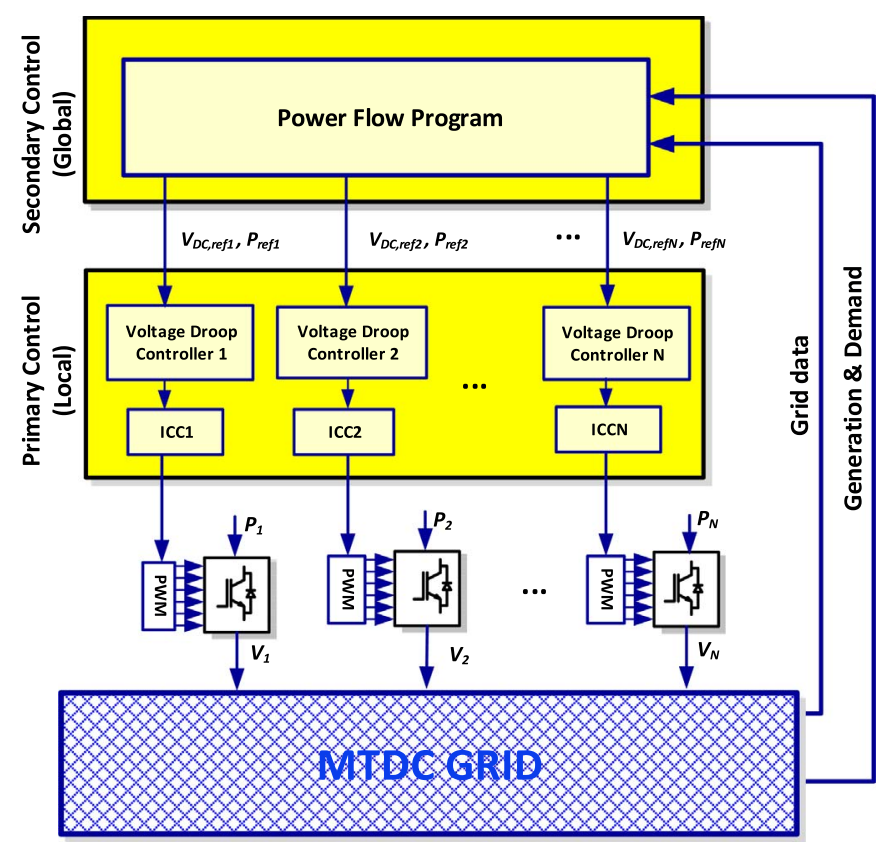

Fig. 13. MTDC grid control structure.

\subsubsection{MTDC grid start-up}

The start-up condition refers to the requirements necessary for the successful black starts of the grid. To be more specific, MTDC grid start-up is associated to the initial direct voltage of the VSC stations when the MTDC grid start from a resting state (i.e., VSC stations are in block mode). When there is no power generation, no power flow should be exit within the MTDC grid. Nevertheless, if the voltage intercepts of the voltage droop characteristics of VSC stations are not identical, there will be an unexpected power flow through the grid even if there is no power injection from stations $[46,48]$. This happens owing to the difference between voltage intercepts $\left(\Delta V_{D C, 0}\right)$ of the VSC voltage-droop characteristics. To avoid such an undesirable phenomenon in MTDC grid, the startup condition can initialize with identical voltage intercept for VSC stations droop characteristics. After grid start-up, the droop characteristics can be somethly re-adjusted according to the planned power sharing and voltage control. 


\subsubsection{Black-start requirements}

Grid black-start is the restoration procedure of the grid from a total or partial black-out. VSC-HVDC technology has the ability to energize and stabilize AC grid during restoration period with its full power capability and few operational restrictions.

An offshore AC grid, powered by VSC-HVDC from mainland may have to be restored after shut down caused by a failure in the HVDC transmission or the offshore AC system. Therefore, it is a necessary that the VSCs can start to work from a dead grid. During an offshore black start, one of the VSC converter stations is connected to the main AC grid and the DC- link capacitors can be charged through this power converter. The DC- link voltage can be hold to a reference value by putting the onshore converter in direct voltage control mode.

Usually, offshore wind farms are not able to establish voltage on their own and their VSC station and DC cables need to be charged to return to normal operation. Implementing a diesel generator in the wind farm can realize the black start procedure of the wind turbine and VSC but this solution would be costly.

Chaudhary et al. [37] presented an MTDC black start procedure by making the receiving end VSC station reverse the normal current direction and work as a rectifier to charge the cable and sending-end VSC station.

Transition from black start control to normal power flow control should be soft, as active and reactive powers on-line measurements will be used as initial active and reactive power settings in the power control mode [37].

\subsubsection{Plug and play characteristics}

The principle of "plug and play" is important. It means that the controls have to be generalized. Considering of MTDC grid dynamics whilst ensuring the interoperability of different VSC stations. This generalized control philosophies should include control specifics that can be different for each VSC station in a multi-vendor set-up, while not compromising the interoperability and overall functionality of MTDC grid. This means that the general control principles of the first installed VSC station should be controlled in such a way, that its control outline would not be subject to changes in the case of adding more VSC stations to the MTDC grid in the future.

\subsubsection{Autonomous control}

Temporary loss of communication between local controls that are located on the VSC station side and the supervisory control layer of the MTDC grid is always a possible threat. Therefore all VSC stations must be able to survive with local measurements to be sure about the stability of the MTDC grid in the absence of communication. This is very similar to AC grid control, where primary frequency controls located on the generation side to ensure about reliable system operation without any need for instantaneous communication to supervisory control. The droop characteristic is allocated to $\mathrm{AC}$ generation units but in MTDC grid will be allocated to the load side converter stations.

\subsubsection{Droop control limitations}

Although, extending the droop control method from the AC grid to MTDC grids is straightforward and several VSC stations can make use of droop control but for assigning the droop slope there is concern about the stability of the VSC stations and overall MTDC grid.

The main obstacle of the droop control is the limitation of powerflow control. For instance, in an offshore grid, steering the harvested energy by the wind farms to a specific bus of the MTDC grid is not possible without communication $[46,48]$.

\subsection{Operational constrains of VSC stations}

To design VSC stations for an MTDC grid certain constraints must be taken into account. All the constraints expressed below should be considered in power flow or optimal power flow routine of AC-DC grid.

\subsubsection{DC link voltage limits}

Voltage at the terminal of the power converters has an up and low limit. The upper limit is determined by the switching components insulation level and the lower limit is determined by limitation of the modulation index of the VSC station. Normally, this limitations are in the range of $\pm 5 \%$ of the nominal direct voltage of DC-link voltage, it means that between 0.95 and $1.05 \mathrm{pu}$ of VSC station nominal voltage.

\subsubsection{Supplied power limits}

Active and reactive power supplied by VSC stations have an upper limits. Active power is limited by the VSC semiconductors current limit, which limits the $\mathrm{AC}$ current and consequently the power (assuming a constant $\mathrm{AC}$ voltage). In addition, active and reactive power are also limited by DC link voltage. However, impact of DC link voltage to limit reactive power is higher than active power. This is analogous to filed current limitation in synchronus generators.

The power limit appears as a hyperbolic curve in the currentvoltage curves and as a straight vertical line in the power-voltage curves.

\subsubsection{DC current limits}

Power converter DC current has only an upper limit considering the current rating of its components. This point appears as a straight vertical line in the IV-characteristics. As the limits depend on the connected DC bus, there is no general answer whether these limits will be reached as the lower voltage limit might be reached before the current limit.

\subsection{MTDC grid operational constraints}

Similar to AC grids there will be a nominal direct voltage for MTDC grid and DC bus voltages should be in the range of $\pm 5 \%$ of nominal direct voltage. The ampacity of the MTDC transmission lines (overhead lines and cables) is another important issue that should be considered as an operational constraint.

\subsection{Protections and DC breakers}

MTDC grids are vulnerable to faults until they are cleared. When DC fault take place, the low impedance of MTDC grid is a tremendous challenge as fault penetration is much faster and deeper than in the case of AC grids [41]. It is crucial to clear the fault before the stored energy in the MTDC grid capacitors is discharged into the fault location. This ensures that the healthy part of the MTDC grid remains connected $[42,43]$. Fault have to be cleared in few milliseconds which is too orders of magnitude shorter than that of AC grids where fault clearing time is in the range of hundreds of milliseconds. Therefore, fast and reliable HVDC breakers are needed, as is the fast fault detection [41].

Significant differences exist between the requirements of $\mathrm{AC}$ and DC breakers principally due to the lack of natural current zero crossing in MTDC grids. In addition to the requirement of very short clearing time, DC breakers have to dissipate considerable amount of energy, which is stored in the MTDC grid [44]. Therefore, in contrast to AC power system, DC breakers are more challenging to build because a mechanism must be included to force current to zero in order to avoid arcing and contact wear [41]. Solid-state breakers is one option, but unacceptably high losses are incurred [41].

In November 2012, ABB launched the world's first hybrid DC breaker [41]. As it can be seen in Fig. 14, the ABB hybrid breaker combines an ultrafast mechanical actuator with IGBT valves. As shown in this figure, the hybrid HVDC breaker consists of two branches. The bypass branch is made by a solid state-based load-commutation switch in series with an ultra-fast mechanical disconnector. The main branch has a solid state-based breaker, which is separated into several sections of series-connected power semiconductors with separate arresters. The 


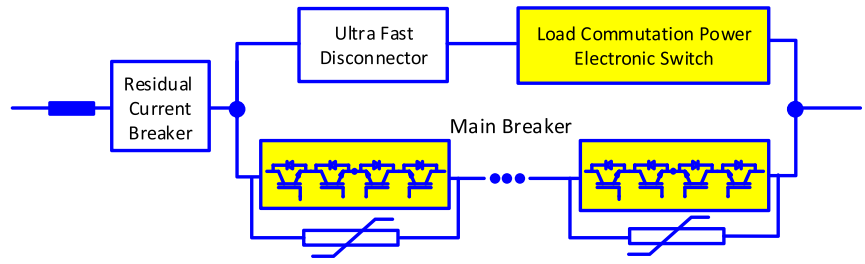

Fig. 14. Illustration of ABB's hybrid HVDC breaker.
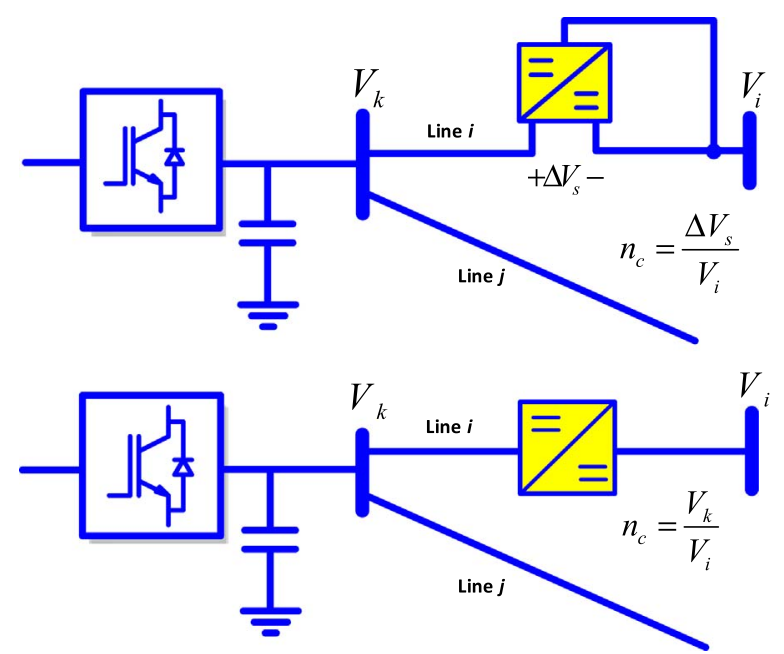

Fig. 15. Two power flow controllers for control of power flow in MTDC grids.

load commutation switch has a low voltage rating.

In the MTD grid normal operation, current flow through the bypass and will not through the main breaker. When a DC fault take place, the main breaker acts and the load-commutations block will open to immediately commutate the current to the main breaker, then the fast disconnector opens. With the mechanical switch in open position, the main breaker open, breaking the current by dissipating the shortcircuit energy in the parallel arresters [41]. Finally, residual disconnecting breaker interrupts the residual current and isolates the faulty transmission line from the MTDC grid.

Currently, maximum breaking current is $16 \mathrm{kA}$ with less than $5 \mathrm{~ms}$ breaking time, including the time delay of the protection system. Hence, the ABB HVDC breaker is adequate for utilization in MTDC grids $[41,45]$. Creation of future MTDC grids strongly depends on the further development of such DC breakers, concerning their rating and cost.

\subsection{Steps towards flexible MTDC grids}

Analogous to the FACTS devices developed in AC grid some power electronic devices should be designed and utilized in MTDC grids to provide flexible control of MTDC grid parameters. So far, the authors of the present paper have used the term "Flexible DC Transmission Systems" or FDCTS as a solution for providing voltage regulation and load flow control in MTDC grids with more flexibility [48].

The FDCTS static devices, which provide either of the functions expressed as follow:

Manipulation of specified DC buses of MTDC grid.

Power flow control and adjustment through the specified transmission lines of MTDC grid.

Currently and based on the power electronics developments, DC/ DC converters (or DC transformers) can provide the abovementioned functions. By adjustment of converter transformation ratio, the voltage on one terminal of the DC/DC converter or the power flow can be regulated. In fact, a DC/DC converter adds a degree of freedom to the control of the MTDC grid. This is similar to what is being carried out by

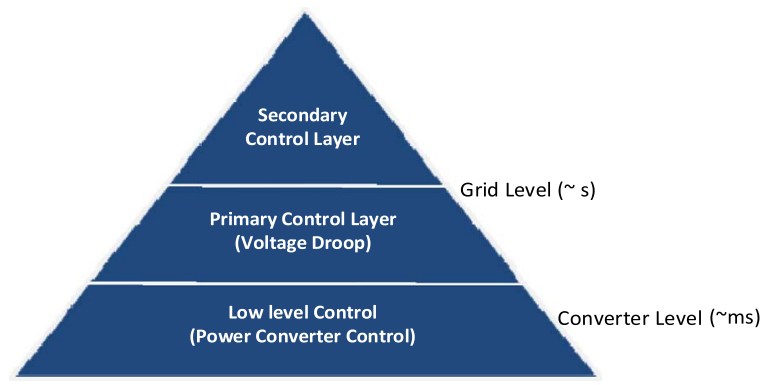

Fig. 16. MTDC grid automation pyramid.

phase-shifting transformers in ac power systems [46]. Fig. 15 shows two power flow controllers, based on the DC/DC converters, for control of power flow in MTDC grids $[46,47,48]$.

\section{Automation pyramid for control of MTDC grids}

All electrical grids DC or AC, requires the implantation of a suitable control structure to ensure stable operation. Nevertheless, the control and operation of MTDC is still an open problem. The full control and operation strategy must include at all time scales involved in the system ranging from milliseconds to some hours [49-51].

The requirement for communication devices on each control level are given such that the delays must be lower than time constants of the controllers on these control levels. An MTDC grid automation pyramid is illustrated in Fig. 16.

\section{Conclusion}

Comprehensive analogies are drawn in this paper when considering emerging MTDC system/grid control and operation in comparison to ac grid control and operation. First, the correspondence between the fundamental parameters of the two systems such as AC grid inertia versus MTDC grid capacitances and frequency versus direct voltage was indicated. Then, the impact of this correspondence to subsequent concepts such as active power transfer or system energy was discussed and summarized.

Later, it was shown that the MTDC grids dynamics analysis can be done in the same way of the ones of AC grids. In view of control analogy, similar control framework of AC grids was proposed to be used for emerging MTDC system/grid. In the primary control layer, the power-frequency droop transformed into a power-voltage droop characteristic. The reason for this choice is the great and well-known advantages of the primary droop-control that allows the generating units parallel connection without obstacle. Unlike the frequency, the direct voltage is not a global parameter in the MTDC grid, but this effect can be suitably compensated by the supervisory control layer.

The MTDC grid secondary control layer that is devoted to power flow needs special attention considering the stochastic behavior of almost all the supposed generating units such as photovoltaic power units and wind power plants in the power flow program or in the optimal power flow program.

\section{Acknowledgements}

This work has been partially supported by the Spanish Ministry of Economy and Competitiveness under the project ENE2014-60228-R. Any opinions, findings and conclusions or recommendations expressed in this material are those of the authors and do not necessarily reflect those of the host institutions or funders.

\section{References}

[1] Wang Peng, Goel L, Liu Xiong, Choo Fook Hoong. Harmonizing ac and dc: a hybrid 
ac/dc future grid solution. IEEE Power Energy Mag 2013;11(3):76-83, [May./ Jun.].

[2] The evolution of HVDC. [online] available:〈http://www.abb.com/ $\rangle$.

[3] Renewables. Global status report [Online]. available: 〈http://www.map.ren21.net/ ; 2015.

[4] EWEA. Wind in power: 2015 European statistics. Technical report, EWEA; 2016

[5] Chowdhury UNA, Yanushkevich A. Power flow analysis of meshed AC-DC super grid, In: Proceedings of the IEEE Eindhoven Power Tech; June 2015, pp.1-6.

[6] Irwin GD, Isaacs A, Woodford D. Simulation requirements for the Atlantic wind multi-terminal VSC offshore wind project. In: Proceedings of the transmission and distribution conference and exposition, pp. 1-4; 2012.

[7] Liang J, Jing T, Bellmunt OG, Ekanayake J, Jenkins N. Operation and control of multi terminal HVDC transmission for offshore wind farms. IEEE Trans Power Del 2011;26(4):2596-604, [Oct.].

[8] Egea-Alvarez A, Bian chi F, Junyent-Ferre A, Gross G, Gomis-Bellmunt OG. Voltage control of multiterminal VSC-HVDC transmission systems for offshore wind power plants: design and implementation in a scaled platform. IEEE Trans Ind Electron 2013;60(6):2381-91, [Jun.].

[9] Bernal-Perez S, Ano-Villalba S, Blasco-Gimenez R, Apari- cio N. Wind power plant control for the connection to multiterminal HVDC links. In: Proceedings of the IEEE energy conversion congress and exposition (ECCE) ;Sep. 2012, pp. 28732879 .

[10] Blau J. Europe plans a North sea grid. IEEE Spectr 2010;47(3):12-3, [Mar.].

11] Van Hertem D, Ghandhari M. Multi-terminal VSC HVDC for the European supergrid: Obstacles,. Renew Sustain Energy Rev 2010;14(9):3156-63, [Dec.].

[12] Bahrman MP, Johnson BK. The ABC of HVDC transmission technologies,. IEEE Power Energy Mag 2007;5(2):32-44, [Mar./Apr.].

[13] Flourentzou N, Agelidis V, Demetriades G. VSC-based HVDC power transmission systems: an overview. IEEE Trans Power Electron 2009;24(3):592-602, [Mar.].

[14] Beerten J, Gomis-Bellmunt O, Guillaud X, Rimez J, van der Meer A, Van Hertem D. Modeling and control of HVDC grids: a key challenge for the future power system. power systems computation conference (PSCC). Wroclaw; 2014

[15] Tielens Pieter, Hertem Dirk Van. The relevance of inertia in power systems. Renew Sustain Energy Rev 2016;55:999-1009, [Mar.].

[16] de Alegria IM, Martin JL, et al. Transmission alternatives for offshore electric power. Renew Sustain Energy Rev 2009;13(5):1027-38, [Jun.].

[17] Rouzbehi K, Gavriluta C, Candela JI, Luna A, Rodriguez .Comprehensive analogy between conventional ac grids and dc grids characteristics. In: Proceedings of the IEEE 39th annual conference industrial electronics society; Nov. 2013, pp. 20042010

[18] Zhang W, Rouzbehi K, Luna A, Gharehpetian GB, Rodriguez P. Multi-terminal HVDC grids with inertia mimicry capability. IET Renew Power Gener 2016. http:// dx.doi.org/10.1049/iet-rpg.2015.0463.

[19] Craciun BI, da Silva R, Teodorescu R., Rodriguez P. Multilink dc transmission for offshore wind power integration. In: Proceedings of the IEEE international symposium on industrial electronics; 2012.

[20] Zhang W, Cantarellas AM, Rocabert J, Luna A, Rodriguez P. Synchronous power controller with flexible droop characteristics for renewable power generation systems. IEEE Trans Sustain Energy 2016. http://dx.doi.org/10.1109/ TSTE.2016.2565059.

[21] Teodorescu R, Liserre M, Rodríguez P. Grid converters for photovoltaic and wind power systems. John Wiley \& Sons; 2011.

[22] Chapman SJ. Electric machinery fundamentals, Fifth edition. McGraw-Hill; 2011.

[23] Fitzgerald AE, Kingsley C, Umans S. Electric machinery. McGraw-Hill; 2003.

[24] Anderson PM, Fouad AA. Power system control and stability. IEEE Press Power Engineering Series \& Wiley-Interscience; 2003.

[25] Rault P, Colas F, Guillaud X, Nguefeu S. Method for small signal stability analysis of VSC-MTDC grids. Power Energy Soc Gen Meet 2012;3:1-7.

[26] Eriksson R, Beerten J, Ghandhari M, Belmans R. Optimizing dc voltage droop settings for ac/dc system interactions. IEEE Trans Power Del 2014;29(1):362-9.

[27] Rouzbehi K, Miranian A, Candela J, Luna A, Rodriquez P. A generalized voltage droop strategy for control of multi-terminal de grids. IEEE Trans Ind Appl 2015;51(1):607-18, [Jan.-Feb.].

[28] Chaudhuri NR, Chaudhuri B. Adaptive droop control for effective power sharing in multi-terminal dc (MTDC) grids. IEEE Trans Power Syst 2013;28(1):21-9, [Feb.].

[29] Yazdani A, Iravanim R. Voltage-sourced converters in power systems: modeling,
Control, and Applications. John Wiley \& Sons; 2010.

[30] Gomez-Exposito A, Conejo A, Canizares C. Electric energy systems analysis and operation. Taylor \& Francis Group; 2009.

[31] Glover JD, Sarma M, Overbye T. Power system analysis and design, Fifth edition. Cengage Learning; 2015.

[32] Pinto RT, Bauer P, Rodrigues SF, Wiggelinkhuizen EJ, Pierik J, Ferreira B. A novel distributed direct-voltage control strategy for grid integration of offshore wind energy systems through MTDC network. IEEE Trans Ind Electron 2013;60(6):2429-41, [Jun.].

[33] Andersen BR, Cartwright P. Control of VSC transmission systems under unbalanced network conditions. IEEE Transmission and distribution conference and exposition PES, vol. 2; 7-12 Sep p 2003.

[34] Rouzbehi K, Miranian A, Luna A, Rodriguez P. Optimized control of multi-terminal dc grids using particle swarm optimization. EPE J 2014;24(2):38-49, [Jun.].

[35] Harnefors L, Bongiorno M, Lundberg S. Input-admittance calculation and shaping for controlled voltage-source converters. IEEE Trans Ind Electron, 2007;54(6):3323-34, [Dec.].

[36] Rouzbehi K, Miranian A, Luna A, Rodriguez P. DC voltage control and powersharing in multi-terminal dc grids based on optimal dc power flow and voltage droop strategy. IEEE J Emerg Sel Top Power Electron 2014;2(4):1171-80, [Dec.]

[37] Chaudhary SK, Teodorescu R, Rodriguez P. Wind farm grid integration using VSC based HVDC transmission-An overview. In:Proceedings IEEE Energy 2030 conference, pp. 1-7; 2008.

[38] Zhang L, Harnefors L, Nee H-P. Interconnection of two very weak ac systems by VSC-HVDC links using power synchronization control. IEEE Trans Power Syst 2011;26(1):344-55, [Feb.].

[39] Yao W, Chen M, Matas J, Guerrero JM, Zhao-ming Q. Design and analysis of the droop control method for parallel inverters considering the impact of the complex impedance on the power sharing. IEEE Trans Ind Electron, 2011;58(2):576-88, [Feb.].

[40] Vasquez JC, Mastromauro RA, Guerrero JM, Liserre M. Voltage support provided by a droop-controlled multifunctional inverter. IEEE Trans Ind Electron 2009;56(11):4510-9, [Nov.].

[41] Häfner J, Jacobson B. Proactive hybrid HVDC breakers - A key innovation for reliable HVDC grids. In: International symposium on integrating supergrids and microgrids, CIGRE, Bologna; 2011.

[42] Yang J, Fletcher JE, Reilly JO. Short-circuit and ground fault analyses and location in VSC-based dc network cables. IEEE Trans Ind Electron 2012;59(10):3827-37, [Oct.].

[43] Franck CM. HVDC circuit breakers: a review identifying future research needs. IEEE Trans Power Del, vol. 26; Apr. 2011, p. 998-1007

[44] Franck CM. HVDC circuit breakers: a review identifying future research needs. IEEE Trans Power Del 2011;26:998-1007, [Apr.].

[45] Weimers L. A european dc super grid-a technology providers view ABB,Brussel; 2011.

[46] Rouzbehi K, Miranian A, Luna A, Rodriguez P. Towards fully controllable multiterminal dc grids using flexible dc transmission systems. IEEE Energy conversion congress and exposition (ECCE); 14-18 Sep. 2014, p. 5312-316.

[47] Rouzbehi K, Candela JI, Luna A, Gharehpetian GB, Rodriguez P. Flexible Control of Power Flow in Multiterminal DC Grids Using DC-DC Converter. IEEE J Emerg Sel Top Power Electron 2016;4(3):1135-44, [Sep.].

[48] Rouzbehi K, Miranian A, Candela JI, Luna A, Rodriguez P. A hybrid power flow controller for flexible operation of multi-terminal DC grids In: Proceedings of the IEEE International conference on renewable energy research and application (ICRERA), p. 550-55, Oct; 2014.

[49] Zhang Weivi, Rouzbehi Kumars, Luna Alvaro, Gharehpetian GB, Rodriguez Pedro. Multi-terminal HVDC grids with inertia mimicry capability. IET Renew Power Gener 2016;10(6):752-60.

[50] Rouzbehi Kumars, Zhang Weiyi, Candela J Ignacio, Luna A, Rodriguez P. Unified Reference Controller for Flexible Primary Control and Inertia Sharing in MultiTerminal VSC-HVDC Grids. IET Gener Transm Distrib, http://dx.doi.org/10.1049/ iet-gtd.2016.0665

[51] Ahmed N, et al. Efficient Modeling of an MMC-Based Multiterminal DC System Employing Hybrid HVDC Breakers. IEEE Trans Power Deliv 2015;30(4):1792-801, [Aug.]. 